\title{
Voxel-based registration of simulated and real patient CBCT data for accurate dental implant pose estimation
}

\author{
António H. J. Moreira ${ }^{*}, \mathrm{a}, \mathrm{b}, \mathrm{c}$, Sandro Queirós ${ }^{\mathrm{a}, \mathrm{b}}$, Pedro Morais ${ }^{\mathrm{a}}$, Nuno F. Rodrigues ${ }^{\mathrm{a}, \mathrm{b}, \mathrm{c}}$, André Ricardo \\ Correia $^{\mathrm{d}}$, Valter Fernandes ${ }^{\mathrm{a}}$, ACM Pinho $^{\mathrm{e}}$, Jaime C. Fonseca ${ }^{\mathrm{b}}$, João L. Vilaça ${ }^{\mathrm{a}, \mathrm{c}}$ \\ ${ }^{a} I C V S / 3 B$ 's - PT Government Associate Laboratory, Braga/Guimarães, Portugal \\ ${ }^{b}$ Algoritmi Center, School of Engineering, University of Minho, Guimarães, Portugal \\ ${ }^{c}$ DIGARC - Polytechnic Institute of Cávado and Ave, Barcelos, Portugal \\ ${ }^{d}$ Faculdade de Medicina Dentária, University of Porto, Porto, Portugal \\ ${ }^{e}$ Mechanical Department, University of Minho, Guimarães, Portugal
}

\begin{abstract}
The success of dental implant-supported prosthesis is directly linked to the accuracy obtained during implant's pose estimation (position and orientation). Although traditional impression techniques and recent digital acquisition methods are acceptably accurate, a simultaneously fast, accurate and operator-independent methodology is still lacking.

Hereto, an image-based framework is proposed to estimate the patient-specific implant's pose using cone-beam computed tomography (CBCT) and prior knowledge of implanted model. The pose estimation is accomplished in a threestep approach: (1) a region-of-interest is extracted from the CBCT data using 2 operator-defined points at the implant's main axis; (2) a simulated CBCT volume of the known implanted model is generated through Feldkamp-Davis-Kress reconstruction and coarsely aligned to the defined axis; and (3) a voxel-based rigid registration is performed to optimally align both patient and simulated CBCT data, extracting the implant's pose from the optimal transformation.

Three experiments were performed to evaluate the framework: (1) an in silico study using 48 implants distributed through 12 tridimensional synthetic mandibular models; (2) an in vitro study using an artificial mandible with 2 dental implants acquired with an i-CAT system; and (3) two clinical case studies.

The results shown positional errors of $67 \pm 34 \mu \mathrm{m}$ and $108 \mu \mathrm{m}$, and angular misfits of $0.15 \pm 0.08^{\circ}$ and $1.4^{\circ}$, for experiment 1 and 2, respectively. Moreover, in experiment 3, visual assessment of clinical data results shown a coherent alignment of the reference implant.

Overall, a novel image-based framework for implants' pose estimation from CBCT data was proposed, showing accurate results in agreement with dental prosthesis modelling requirements.
\end{abstract}

Keywords: dental prosthetics; cone-beam CT simulation; voxel-based rigid registration; computer-aided modelling

\section{INTRODUCTION}

The medical treatment of dental replacement has been significantly improved after the diffusion of osseointegrated implants to support dental prosthesis. Nowadays, different techniques are available for manufacturing and assembling these biomechanical systems, commonly based on a metallic bridge structure supported by a series of screws implanted in the edentulous patient's jaw. Traditionally, an impression procedure is employed, where various steps directly influence the fit between the prosthesis and the implants, and ultimately affect the prosthesis long-term success ${ }^{1}$. In fact, factors such as the impression material, impression copings, implant position/orientation or the dentist experience affect the accuracy of the implant's pose estimation ${ }^{2}$. Thus, obtaining an absolute passive fit is still nearly impossible, especially in partially or completely edentulous patients. While an agreement over clinical acceptability has never been reached, a misfit under $120 \mu \mathrm{m}$ is usually accepted given that it does not lead to future implant complications ${ }^{3}$, either of mechanical or biological origin.

*antoniomoreira@ecsaude.uminho.pt; phone +351 253604 891; fax +351 253604 809; www.besurg.com

\footnotetext{
Medical Imaging 2015: Computer-Aided Diagnosis, edited by Lubomir M. Hadjiiski, Georgia D. Tourassi,
} Proc. of SPIE Vol. 9414, 94143H - (c) 2015 SPIE · CCC code: 1605-7422/15/\$18 - doi: 10.1117/12.2082806 
To ease the procedure and overcome the inherent accuracy problems of impression techniques, recent developments within optical devices (3D intra-oral scanners) and electromagnetic systems have been made ${ }^{4-6}$. Although optical devices improve patient acceptance, reduce possible distortion of impression materials and increase efficacy, they are an additional expense to the clinic and are operator-dependent with accuracies near the traditional impression procedure ${ }^{7,8}$. In addition, some require additional powder to improve the reflection in the mucosa region, complicating the procedure.

In dental clinical practice, cone-beam computed tomography (CBCT) is a commonly imaging modality used for threedimensional acquisitions of the oral and maxillofacial region with high resolution and low radiation ${ }^{9}$. Commonly applied in a large variety of dental applications, such as implant planning, orthodontics and endodontics, it also provides valuable information on the implants' pose inside the patient's jaw in an easy, non-invasive way. However, to the author's best knowledge, it was never used in a post-operative stage to estimate the position and orientation of the implants and, from this information, design the implant-supported prosthesis.

To overcome the limitations of traditional and optical techniques, and taking advantage of current CBCT systems, in this work, we present an image-based framework to optimally estimate the patient-specific implant's pose from postoperative $\mathrm{CBCT}$ data and from prior knowledge of implanted model. With this framework, an operator-independent, accurate and fast methodology is targeted, creating an opportunity to further ease computer-aided modelling of superstructure prosthesis, while using commonly available imaging technologies.

\section{METHODS}

Accurate implant's pose estimation is essential for computer-aided modelling of a superstructure prosthesis. Aiming to ease this process, the fundamental concept of the proposed framework is to compute the geometrical transformations needed to place 3D implant reference models over the implants in the patient's CBCT data, thus describing their relative pose (Figure 1). The framework workflow relies on a volumetric simulation of a reference implant model through a conebeam reconstruction algorithm, approximating it to the one in the patient's CBCT data. Subsequently, using specific shape information present in the reference model, both simulated and real volumes are registered allowing the implant's pose extraction from the optimal transformation. A description of this process is provided in the following sub-sections.

\subsection{User Input and ROI Extraction}

Since searching for implants in full CBCT volumes is a computationally expensive approach, we propose to semiautomatically extract, prior to the pose estimation, small regions of interest (ROI) centered on the implants of interest. Such ROI extraction also easies the subsequent steps by decreasing the possibility for local minima during the registration step and thus providing a more robust and refined result. To this end, two points per implant (at the implant's base and tip) must be provided by the expert, describing the implant's main axis. This information is used to extract a fixed ROI volume with $20 \mathrm{~mm}^{3}$ of size at the central position between the two points (Figure 1A). This region was defined as a $20 \mathrm{~mm}^{3}$ volume since it covers almost every implant in market (ranging from $6 \mathrm{~mm}$ up to $17 \mathrm{~mm}$ in length). In addition, the user-defined axis is also exported to be used in the subsequent step of the approach (Figure 1A, blue arrow).

\subsection{Implant CBCT Simulation}

CBCT is a well-known system for acquiring 3D volumes of the oral and maxillofacial region with high resolution. Nevertheless, the detail achieved in the output volume is obviously limited by its spatial resolution, which in the specific case of dental implants leads to the distortion of their characteristic features (i.e. size, spiral count and pitch) and ultimately hampers pose estimation. With this in mind, we propose to reconstruct a volume of the implant model, using similar characteristics to the acquisition system, in order to mimic the implanted one. Such simulated volume adds prior knowledge to the framework by closely describing the appearance of the implant to be detected.

Starting with a triangular-polygon mesh of the real implanted model, a ray intersection method $^{10}$ is used to obtain a binary volume of the implant of interest (Figure 1B). This method ray-traces the implant mesh in the $\mathrm{X}, \mathrm{Y}$ and $\mathrm{Z}$ directions, with the overall result being the combination of their results (Figure 2).

Subsequently, a CBCT reconstruction algorithm is used to obtain the final simulated volume. To this end, the binary volume is projected in 360 slices with $1^{\circ}$ step and reconstructed (Figure 2) using the Feldkamp-David-Kress algorithm on a graphics processing unit (GPU-based) cone-beam CT reconstruction software (OpenRTK) ${ }^{11}$. 


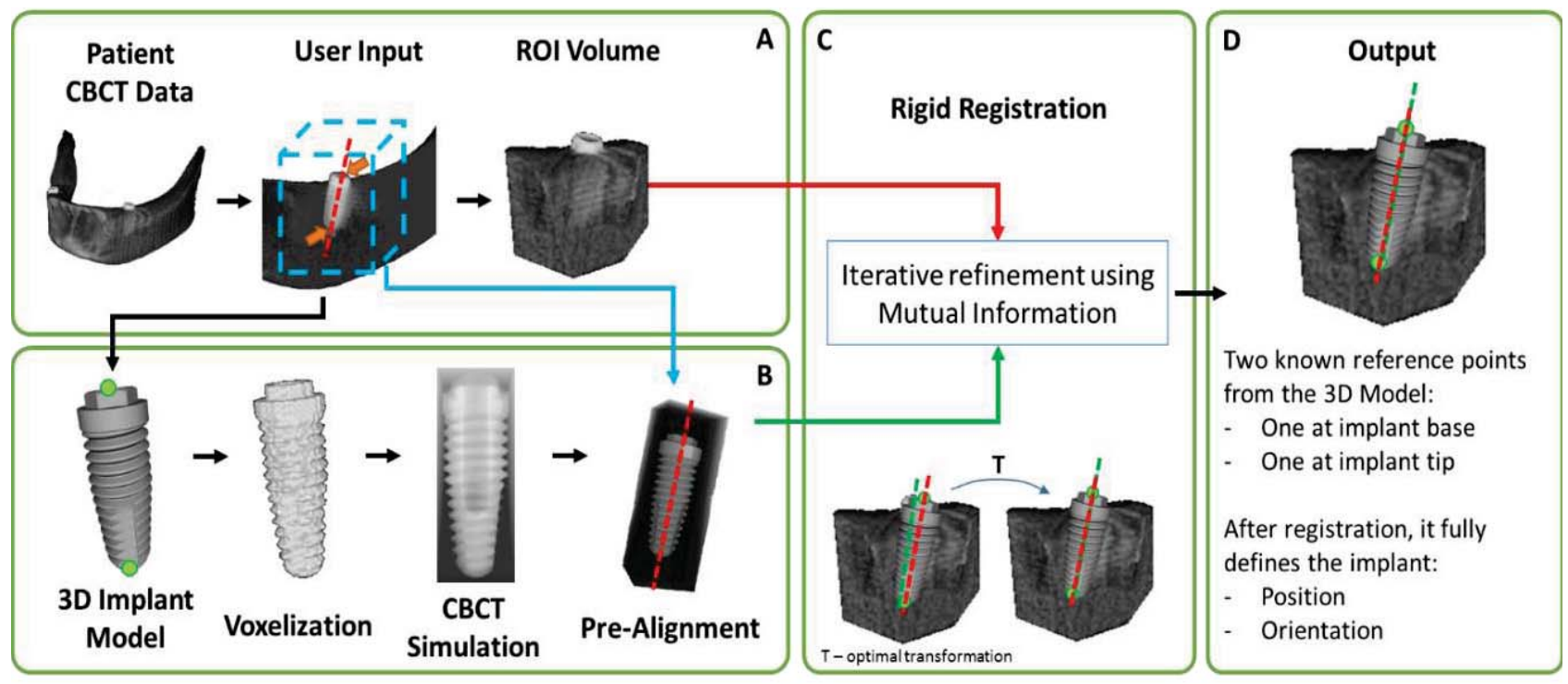

Figure 1: Framework workflow. (A) CBCT data input and manual selection of 2 points at implant's base and tip (orange arrows) to extract a ROI centered in the implant of interest. (B) Implant type identification (black arrow), followed by a mesh voxelization and a volumetric simulation with cone-beam CT characteristics. The resulting volume is pre-aligned using the operator-defined axis (blue arrow). (C) Alignment refinement using rigid registration between the simulated implant volume (green arrow) and the patient's ROI volume (red arrow). (D) Two reference points (green dots) from the 3D implant model are mapped to the patient CBCT data, estimating the implant's pose (red dashed line). Note that the green dashed line represents the perfect pose for the implant of interest.

\subsection{Voxel-Based Registration}

Given the operator-defined points, an initial pre-alignment (defined by the average position between the two points and their orientation) places the reconstructed volume over the real CBCT data. Then, a refinement process is applied to optimally align both simulated and real CBCT volumes and thus obtain an accurate pose estimation. This is achieved through a rigid registration strategy, where an iterative alignment maximizes the similarity between the two volumes. Note that the presence of local implant features, similar in both real and simulated volumes, steers the result towards an optimal superposition, thus significantly decreasing the pose estimation inaccuracies.

In the current implementation, the Elastix framework was employed ${ }^{12}$, with a similarity metric based on Mutual Information, B-Spline interpolation and Adaptative Stochastic Gradient Optimizer (Figure 1C). The final isotropic grid spacing was set to 1 voxel and 4 scales were used during the multi-resolution approach (halving the spacing in the threedimensional direction in every scale).

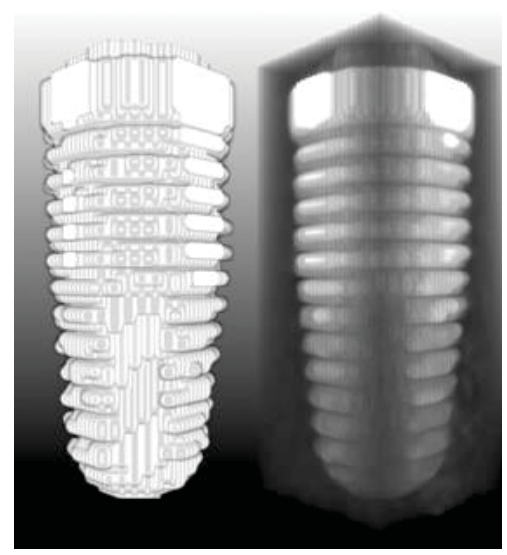

Figure 2: Example of voxelized implant volume at left, and simulated implant volume at right, both at $0.2 \mathrm{~mm}$ resolution. 
Finally, the optimal transformation matrix is obtained by combining the initial pre-alignment (described in section 2.1) and the subsequent refinement transformation (presented in section 2.3), describing how the implant 3D mesh model is fitted into the real CBCT data. Using such transformation, two points previously defined in the triangular-polygon mesh, at the implant's base and tip, are mapped to the real patient CBCT volume world, thus describing the final implant's pose.

\section{EXPERIMENTS}

Three experiments (in silico, in vitro and clinical case studies) were used to evaluate the proposed framework.

In the in silico study, 48 implants of three different brands (Wital, MIS and ABDental) were placed at known locations in twelve 3D simulated models of a human maxilla (4 models with 2 implants, 4 models with 4 implants and 4 models with 6 implants). Each composed virtual model (3D triangular polygon mesh of a maxilla including implants) was simulated through a cone-beam CT reconstruction algorithm (as previously described in section 2.2). To assess the misfit sensitivity to the image resolution, each model was reconstructed at 5 different resolutions (from $0.1 \mathrm{~mm}$ up to $0.5 \mathrm{~mm}$ ) and the proposed framework applied in each one. Moreover, to analyze the potential beneficial effect of using the implant CBCT simulation to mimic the implant's appearance, a comparison between simulated and voxelized volumes (used during the registration step) was performed in terms of distance and angle error.

Regarding the in vitro study, one standard composite artificial mandible from Promedicus (Brzoskwiniowa 11, Poland), with 2 MIS Seven implants (MIS Implants Technologies Ltd), was scanned with an i-CAT CBCT system at $0.125 \mathrm{~mm}$ resolution and used to evaluate our framework (Figure 3A). The implant's pose ground-truth was measured with a Wenzel $3 \mathrm{D}$ coordinate measuring machine (CMM) LH 54 with $2 \mu \mathrm{m}$ of accuracy. Then, the inter-implant distance and angle error between the estimated pose and the real model were measured.

Lastly, two clinical case studies were used to assess the feasibility of the proposed approach in a clinical setting. Both $\mathrm{CBCT}$ volumes were acquired with an i-CAT CBCT system at $0.3 \mathrm{~mm}$ of resolution (Figure 3B-C). The feasibility result is achieved through visual inspection, comprehending two key aspects: a) volume overlay, and b) main axis's angle difference between the estimated pose and the ground truth.

\section{RESULTS}

Regarding the in silico study, Table 1 summarizes the position and angular errors for voxelized and simulated implant references at different image resolutions. Different error rates are perceptible through decrease of resolution (from $0.1 \mathrm{~mm}$ up to $0.5 \mathrm{~mm}$ ) when using the simulated or the voxelized model. Note that at the highest resolution $(0.1 \mathrm{~mm})$ both simulated and voxelized models achieve a similar accuracy, although from $0.2 \mathrm{~mm}$ up to $0.5 \mathrm{~mm}$ the simulated model shows the best positional and angular accuracy.

Table 2 presents the implant's shape influence in the accuracy of the proposed framework by assessing the misfits for the three brands independently. In this case, a paired t-test was performed between implant's brands showing significant differences, particularly in positional accuracy.

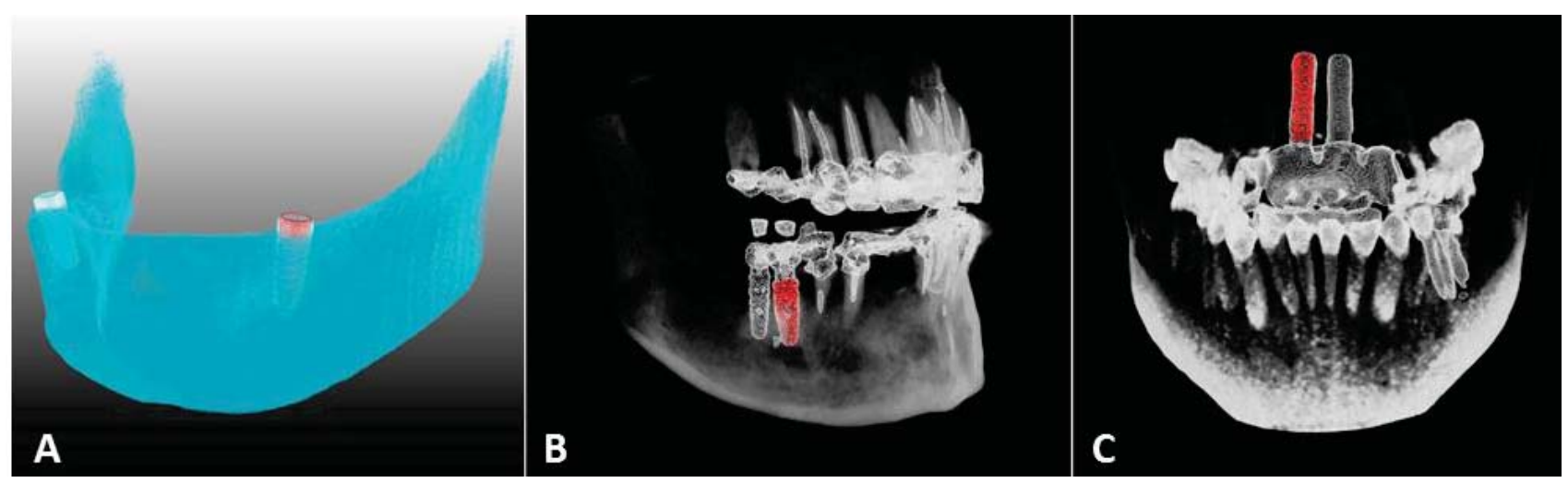

Figure 3: Visual assessment of in vitro experiment and clinical case studies. A - In vitro evaluation: implant model in red, human mandible in blue and implants in white. B and C - Implant models (red) overlaid in patient CBCT data. 
Table 1: Position and angular errors for voxelized and simulated implant references at different resolutions $(\mu \pm \sigma)$.

\begin{tabular}{|c|c|c|c|c|c|c|c|c|c|c|}
\hline & \multicolumn{2}{|c|}{$0.1 \mathrm{~mm}$} & \multicolumn{2}{|c|}{$0.2 \mathrm{~mm}$} & \multicolumn{2}{|c|}{$0.3 \mathrm{~mm}$} & \multicolumn{2}{|c|}{$0.4 \mathrm{~mm}$} & \multicolumn{2}{|c|}{$0.5 \mathrm{~mm}$} \\
\hline & Dist $(\mu \mathrm{m})$ & $\theta\left({ }^{0}\right)$ & Dist $(\mu \mathrm{m})$ & $\theta\left({ }^{\circ}\right)$ & Dist $(\mu \mathrm{m})$ & $\theta\left({ }^{\circ}\right)$ & Dist $(\mu \mathrm{m})$ & $\theta\left({ }^{\circ}\right)$ & Dist $(\mu \mathrm{m})$ & $\theta\left({ }^{\circ}\right)$ \\
\hline Simulated & $67 \pm 34$ & $0.15 \pm 0.08$ & $152 \pm 61$ & $0.24 \pm 0.14$ & $215 \pm 101^{a}$ & $0.43 \pm 0.28$ & $306 \pm 127^{a}$ & $0.57 \pm 0.38$ & $447 \pm 134^{a}$ & $0.69 \pm 0.35^{\mathrm{a}}$ \\
\hline Voxelized & $63 \pm 26$ & $0.13 \pm 0.06$ & $174 \pm 45$ & $0.26 \pm 0.14$ & $280 \pm 85$ & $0.50 \pm 0.26$ & $447 \pm 88$ & $0.76 \pm 0.44$ & $543 \pm 145$ & $1.05 \pm 0.5$ \\
\hline
\end{tabular}

${ }^{a} p<0.05$, paired t-test against voxelized reference.

Table 2: Position and angular errors for different implant models using voxelized and simulated implant references (image resolution of $0.1 \mathrm{~mm}, \mu \pm \sigma)$.

\begin{tabular}{|c|c|c|c|c|}
\hline \multirow{2}{*}{ Type } & \multicolumn{2}{|c|}{ Simulated } & \multicolumn{2}{|c|}{ Voxelized } \\
\hline & Dist $(\mu \mathrm{m})$ & $\theta\left({ }^{\circ}\right)$ & Dist $(\mu \mathrm{m})$ & $\theta\left({ }^{\circ}\right)$ \\
\hline ABDental & $89 \pm 39^{c}$ & $0.12 \pm 0.06$ & $78 \pm 29^{b}$ & $0.12 \pm 0.04$ \\
\hline MIS & $65 \pm 25$ & $0.17 \pm 0.09$ & $43 \pm 18^{\mathrm{a}, \mathrm{c}}$ & $0.11 \pm 0.06$ \\
\hline Wital & $48 \pm 21^{\mathrm{a}}$ & $0.15 \pm 0.09$ & $69 \pm 15^{b}$ & $0.13 \pm 0.08$ \\
\hline
\end{tabular}

In the in vitro study, an error of $108 \mu \mathrm{m}$ and $1.41^{\circ}$ of angular misfit was obtained for the relative pose between implants. Finally, the results for the two clinical case studies presented an acceptable overlay between real implant data and simulated volumetric data, in both volume and main axis angle (Figure 3B-C).

\section{DISCUSSION}

Regarding the in silico study, Table 1 shows an improvement in accuracy for higher spatial resolutions of the voxelized and simulated volumes. Note that both techniques presented similar positional accuracy $(67 \pm 34 \mu \mathrm{m}$ vs $63 \pm 26 \mu \mathrm{m})$ and lowest angular misfit $\left(0.15 \pm 0.08^{\circ}\right.$ vs $\left.0.13 \pm 0.06^{\circ}\right)$ at the highest resolution (voxel size of $0.1 \mathrm{~mm}$ ). Furthermore, in both cases, the rate of change over the five tested resolutions was appreciable. The disparity in positional accuracy between voxelized and simulation models achieved its highest difference at $0.4 \mathrm{~mm}$ of resolution (Table 1), with simulated references achieving better results. Considering the angular misfit, the maximum angular discrepancy was found at 0.5 $\mathrm{mm}$ of resolution (Table 1), with simulated references' accuracy surpassing voxelized one. The current results can be explained with the increase on image appearance detail available inside the implant volume when applying the CBCT simulation, which can be easily observed when reducing the voxel size from $0.5 \mathrm{~mm}$ to $0.2 \mathrm{~mm}$.

In relation to Table 2, it is possible to identify statistically significant differences between implant's brands for accurate position estimation. The results suggest that the shape, size and the unique characteristic of each implant (in particular the helix pitch and outline) can influence the overall accuracy of this framework. However, the results are kept within clinical acceptable value ranges (a value for passive fit between implants and the supporting structure is still undefined, misfit below $120 \mu \mathrm{m}$ have been reported in the past ${ }^{3}$ ).

Regarding the in vitro study (Figure 3A), a relative inter-implant distance error of $108 \mu \mathrm{m}$ and $1.41^{\circ}$ of angular misfit was reported, in line with the in silico results. Although the angular misfit is higher, this can be associated with the CMM acquisition protocol or uncalibrated geometrical parameters of the CBCT system. The later may generate an uneven volume and therefore introduce misfit errors, which should be confirmed in a future work through the acquisition of additional CBCT scans in supervised conditions.

Lastly, the two clinical case studies presented an acceptable visual agreement (Figure 3B-C), but the lack of implants positional and angular ground-truth (through the availability of the digital prosthesis model) invalidated a more adequate analysis.

\section{CONCLUSIONS}

In this study, a novel image-based framework was proposed to accurately assess the implant's pose directly from CBCT data based on prior knowledge of the implants in the patient jaw. Overall, the results $\left(67 \pm 34 \mu \mathrm{m}\right.$ and $\left.0.15 \pm 0.08^{\circ}\right)$ shown that current $\mathrm{CBCT}$ technology fused with our proposed image-based framework can achieve the accuracy demands of 
dental prosthesis modelling. In fact, the advantages of the image-based framework are two-fold: 1) fast and accurate pose estimation; 2) operator-independent methodology. Additionally, this approach can boost CBCT usage as an alternative to the traditional procedure and overcome the inherent accuracy problems of impression techniques.

However, the concern for a second exam with additional CBCT radiation dosage may limit its usage in a clinical environment. Currently, CBCT already presents a low radiation dosage when compared to other CT machines, however the final volume resolution is influenced by the time of exposure and/or field-of-view. Despite this fact, the observed accuracy at lower resolutions $(0.2 \mathrm{~mm}-0.5 \mathrm{~mm})$ could still potentiate the use of this framework, since it is possible to minimize radiation exposure during image acquisition without losing overall pose estimation accuracy.

Currently, we are extending both in vitro and clinical evaluations for cases with multiple implant connections in edentulous patients. As future work, combining mucosa's surface with accurate implant's location can further ease computer-aided modelling of superstructure prosthesis.

\section{ACKNOWLEDGEMENTS}

This work has been supported by FCT - Fundação para a Ciência e Tecnologia in the scope of the Ph.D. grant SFRH/BD/68270/2010, SFRH/BD/93443/2013 and the project EXPL/BBB-BMD/2146/2013.

We also gratefully acknowledge the assistance of Pedro Macedo, Senior Multimedia Technician from Dr. Manuel Neves Clinic and Technical CAD-CAM Laboratory InoveSmile, Porto, Portugal.

\section{REFERENCES}

[1] Lee, H., So, J. S., Hochstedler, J. L.., Ercoli, C., "The accuracy of implant impressions: a systematic review.," J. Prosthet. Dent. 100(4), 285-291 (2008).

[2] Sorrentino, R., Gherlone, E. F., Calesini, G.., Zarone, F., "Effect of implant angulation, connection length, and impression material on the dimensional accuracy of implant impressions: an in vitro comparative study.," Clin. Implant Dent. Relat. Res. 12 Suppl 1, e63-e76 (2010).

[3] Sahin, S.., Cehreli, M. C., "The significance of passive framework fit in implant prosthodontics: current status.," Implant Dent. 10(2), 85-92 (2001).

[4] Van der Meer, W. J., Andriessen, F. S., Wismeijer, D.., Ren, Y., "Application of intra-oral dental scanners in the digital workflow of implantology.," PLoS One 7(8), e43312 (2012).

[5] Moreira, A. H. J., Queiros, S., Rodrigues, N. F., Pinho, A. C. M., Fonseca, J. C.., Vilaca, J. L., "Electromagnetic tracker feasibility in the design of a dental superstructure for edentulous patients," 2014 IEEE Int. Symp. Med. Meas. Appl., 1-6, IEEE (2014).

[6] Moreira, A. H. J., Queirós, S., Rodrigues, N. F., Pinho, A., Fonseca, J. C.., Vilaça, J. L., "An electromagnetic tracker system for the design of a dental superstructure," III Int. Conf. Biodental Eng. (2014).

[7] Lee, S. J.., Gallucci, G. O., "Digital vs. conventional implant impressions: efficiency outcomes.," Clin. Oral Implants Res. 24(1), 111-115 (2013).

[8] Giménez, B., Ozcan, M., Martínez-Rus, F.., Pradíes, G., “Accuracy of a Digital Impression System Based on Active Wavefront Sampling Technology for Implants Considering Operator Experience, Implant Angulation, and Depth.," Clin. Implant Dent. Relat. Res. (2013).

[9] Scarfe, W. C., Li, Z., Aboelmaaty, W., Scott, S. A.., Farman, A. G., "Maxillofacial cone beam computed tomography: essence, elements and steps to interpretation.," Aust. Dent. J. 57 Suppl 1, 46-60 (2012).

[10] Patil, S.., Ravi, B., "Voxel-based Representation, Display and Thickness Analysis of Intricate Shapes," Ninth Int. Conf. Comput. Aided Des. Comput. Graph., 415-422, IEEE (2005).

[11] Rit, S., Vila Oliva, M., Brousmiche, S., Labarbe, R., Sarrut, D.., Sharp, G. C., "The Reconstruction Toolkit (RTK), an open-source cone-beam CT reconstruction toolkit based on the Insight Toolkit (ITK)," J. Phys. Conf. Ser. 489(1), 012079 (2014).

[12] Klein, S., Staring, M., Murphy, K., Viergever, M. A.., Pluim, J. P. W., "elastix: a toolbox for intensity-based medical image registration.," IEEE Trans. Med. Imaging 29(1), 196-205 (2010). 